\title{
Effect of Batch Melting Temperature and Raw Material on Iron Redox State in Sodium Silicate Glasses
}

\author{
Bahman Mirhadi and Behzad Mehdikhani \\ Engineering department, Imam Khomeini International University, Qazvin, Iran \\ (Received August 11, 2010; Revised October 18, November 20, 2010; Accepted December 29, 2010)
}

\begin{abstract}
In this study, the redox state of iron in sodium silicate glasses was varied by changing the melting conditions, such as the melting temperature and particle size of iron oxide. The oxidation states of the iron ion were determined by wet chemical analysis and UV-Vis spectroscopy methods. Iron commonly exists as an equilibrium mixture of ferrous ions, $\mathrm{Fe}^{2+}$, and ferric ions $\mathrm{Fe}^{3+}$. In this study, sodium silicate glasses containing nanoparticles of iron oxide $(0.5 \% \mathrm{~mol})$ were prepared at various temperatures. Increase of temperature led to the transformation of ferric ions to ferrous ions, and the intensity of the ferrous peak in $1050 \mathrm{~nm}$ increased. Nanoparticle iron oxide caused fewer ferrous ions to be formed and the $\frac{\mathrm{Fe}^{2+}}{\mathrm{Fe}^{3+}}$ equilibrium ratio compared to that with
micro-oxide iron powder was lower.
\end{abstract}

Key words : Nanoparticles, Iron oxide, UV-Vis spectroscopy, Wet chemical analysis

\section{Introduction}

$\mathrm{T}$ he majority of commercial green glass contains iron oxide $^{1,2)}$ because it is made with raw materials with low purity. Iron absorption peaks in the visible region lead to the development of green coloring. ${ }^{2)}$ Any effect of iron oxide in colorless glasses is not visible easily. To investigate its effects, it is better to consider it from the viewpoint of thickness because the vision route in this direction is longer. ${ }^{3)}$ Controlling the iron oxidation state in commercial glasses for the development of special colors or elimination of produced colors as a result of iron oxide is very important. ${ }^{4)}$ The absorption of infrared rays by glasses containing iron is also important for the protection of humans from harmful effects. ${ }^{1)}$

The effects of iron on the optical properties of glass have been studied widely by many researchers. ${ }^{5-8)}$ Because iron has two redox states, $\mathrm{Fe}^{2+}$ and $\mathrm{Fe}^{3+}$, and these states have different colors, control of the redox condition during melting is important in adjusting the transmittance of glass in the wavelength regions of UV, visible light, and IR. ${ }^{6}$ When melted glass is placed in a reductive environment, the content of $\mathrm{Fe}^{2+}$ increases, and the glass turns bluish green because $\mathrm{Fe}^{2+}$ has a broad absorption band centered at $1050 \mathrm{~nm} .^{7,8)}$ This absorption band is utilized in IR-cut glass such as cold-filtered glass which is produced under reductive conditions. When melted glass is placed in an oxidative environment, the iron in the glass is oxidized to the $\mathrm{Fe}^{3+}$

${ }^{\dagger}$ Corresponding author : Behzad Mehdikhani

E-mail : beh_mehdikhani@yahoo.com, mirhadi_bahman@yahoo.com Tel : +989126417516 Fax : +982413230496 state and produces a yellowish color due to an absorption band which shows strong absorption below $380 \mathrm{~nm}$ that tails off towards longer wavelengths up to about $450 \mathrm{~nm} .^{9,10)}$ Such glass containing $\mathrm{Fe}^{3+}$ in large amounts is used as UVcut glass. In particular, changes in melting temperature result in varying amounts of ferrous and ferric ions in glass. ${ }^{6)}$ Controlling the redox state of Fe ions in glass is therefore important for optical applications.

In this study, sodium silicate glass batches containing a specific amount of nanoparticle iron oxide were prepared and melted at various temperatures. Their UV-Vis spectra were traced, and the amount of ferrous ions were determined by wet chemical analysis as in the Joung method. Based on the results, the influence of nanoparticle iron oxide and melting temperature on the intensity of absorption peaks was discussed.

\section{Experimental Procedure}

In these experiments, the batches were mixed from reagent grade raw materials; $\mathrm{SiO}_{2}$ and $\mathrm{Na}_{2} \mathrm{Co}_{3}$. As an iron oxide additive, nano-powder iron oxide with a grain size of $6 \mathrm{~nm}$ and purity of 99/9\% made by GmbH Plasma Chem Company was used in these experiments. Chemical compositions of the glasses and batch melting temperatures are summarized in Table 1. Glass batches were melted in an alumina crucible and a VECSTAR kiln with air atmosphere. The rate of heating for all samples by this kiln was $5^{\circ} \mathrm{C} / \mathrm{min}$ at room temperature. The loading of the batches into the crucible was done at temperatures between 900 and $950^{\circ} \mathrm{C}$. These temperatures should not be higher because it causes a substantial volatilization of reagents. After an entire batch was pre-melted and sintered in the crucible, the tem- 
Table 1. Chemical Composition of Glass and Batch Melting Temperature

\begin{tabular}{ccccc}
\hline sample no & $\mathrm{SiO}_{2}$ & $\mathrm{Na}_{2} \mathrm{O}$ & $\mathrm{Fe}_{2} \mathrm{O}_{3}$ & Melting temperature ${ }^{\circ} \mathrm{C}$ \\
\hline 1 & & & & 1350 \\
2 & 66.3 & 33.2 & 0.5 & 1450 \\
3 & & & & 1550 \\
4 & & & & 1650 \\
\hline
\end{tabular}

perature was increased stepwise to the melting temperature. The processing temperature was varied from 1350 to $1650^{\circ} \mathrm{C}$ and maintained for 2 to $2 \frac{1}{2} \mathrm{~h}$. The lasting period is very important because 1 ) the melting temperature (also marked as the equilibrium temperature) is later used as a temperature for thermodynamic calculations, 2) bubbles must be removed from the glass melts which could disturb the optical absorption measurements and 3) glass melts are homogenized in the whole crucible. After this period, the glass was quickly cast at the same temperature in a preheated $\left(\mathrm{T}=450^{\circ} \mathrm{C}\right)$ stainless steel mold with dimensions of $2 \mathrm{~cm} \times 2 \mathrm{~cm} \times 2 \mathrm{~mm}$ to avoid temperature-caused inhomogeneity. It was then cooled in a preheated furnace to ambient temperature using a cooling rate of $30 K . h^{-1}$. After grinding and polishing, the absorption spectra of the glass samples were recorded by a Camspec Double (Beam UV-VIS $350 \mathrm{~m}$ ) spectrophotometer in an optical range from 200 to $1100 \mathrm{~nm}$. Wet chemical analysis was performed according to the Jeoung method. ${ }^{12)}$

\section{Results and Discussion}

Fig. 1 shows the ultraviolet-visible spectra of sodium silicate glasses containing iron oxide at various temperatures. The absorbance for $\mathrm{Fe}^{3+}$ produces peaks at about 380, 420, and $435 \mathrm{~nm}$ on the UV absorption edge, while $\mathrm{Fe}^{2+}$ indicates a broad band peak at around $1050 \mathrm{~nm}$. The optical peaks due to $\mathrm{Fe}^{3+}(350 \sim 500 \mathrm{~nm})$ were identical in all the melted samples as shown in Fig. 2. As the temperature increased, the intensity of the absorption peak at $1050 \mathrm{~nm}$ also increased. This result means that the amount of ferrous ions had increased. The amount of increase or decrease in ferric and ferrous ions with increasing temperature was

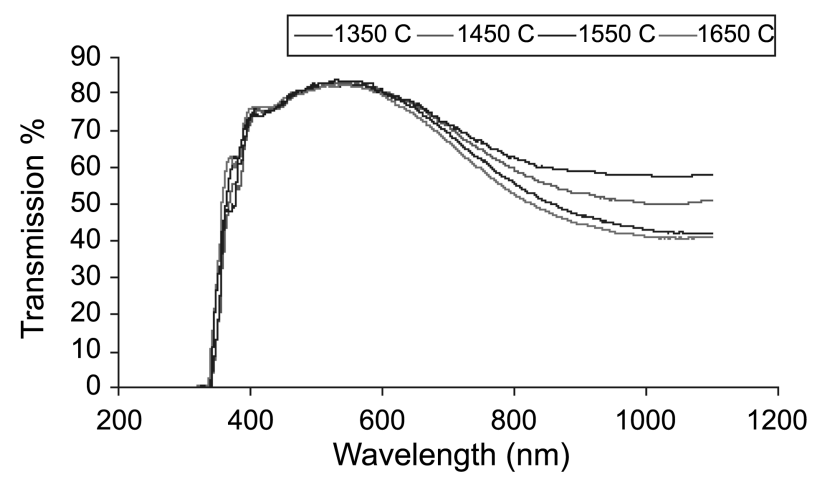

Fig. 1. Effect of melting temperature on the optical absorption of iron-sodium silicate glasses.

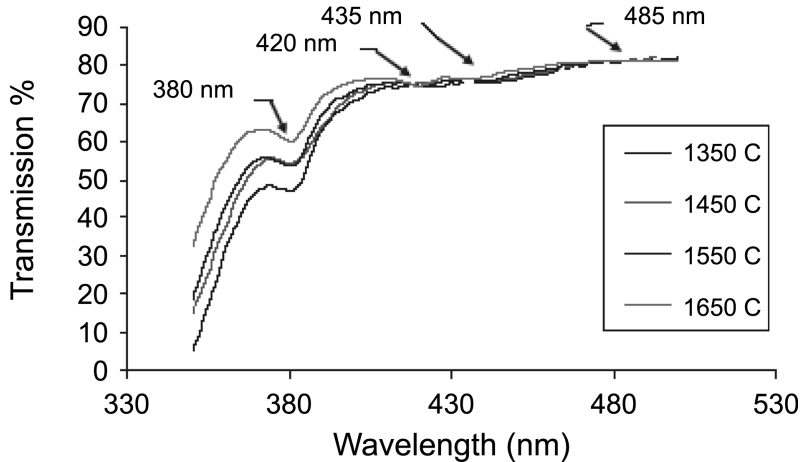

Fig. 2. Effect of melting temperature on the optical absorption of iron-sodium silicate glasses. $350 \sim 500 \mathrm{~nm}$ wavelength regions.

Table 2. Amount of $\mathrm{Fe}^{3+}$ and $\mathrm{Fe}^{2+}$ Ions Obtained by Wet Chemical Analysis

\begin{tabular}{cccc}
\hline sample $\left({ }^{\circ} \mathrm{C}\right)$ & $F e^{3+} W t \%$ & $F e^{2+} W t \%$ & $\frac{F e^{2+}}{F e^{3+}}$ \\
\hline 1350 & 0.93 & 0.12 & 0.13 \\
1450 & 0.88 & 0.17 & 0.19 \\
1550 & 0.82 & 0.23 & 0.28 \\
1650 & 0.81 & 0.24 & 0.29 \\
\hline
\end{tabular}

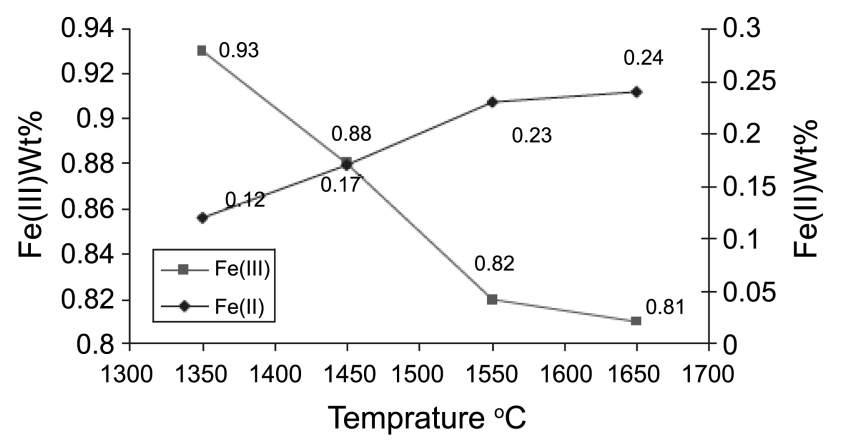

Fig. 3. Effect of increasing temperature on changing amounts of ferric and ferrous ions in iron-sodium silicate glasses.

determined by wet chemical analysis, and the results are shown in Table 2 and Fig. 3. Increase of temperature led to a slight change in the chemical composition of glass. By putting a bonnet on the crucible during melting, this change was minimized. At low temperatures before the formation of the melt, reaction between oxygen in the atmosphere and species in the raw material occurs easily, and this affects the redox state of the species and the oxygen activity in the melt. Oxygen is produced in the melt at elevated temperature if the material contains $\mathrm{Fe}^{3+}$ as a result of a reaction with $\mathrm{O}^{2-}$, which is abundantly present in the material. The equation can be written as in $^{6)}$ as

$$
4 \mathrm{Fe}^{3+}+2 \mathrm{O}^{2-} \longleftrightarrow 4 \mathrm{Fe}^{2+}+\mathrm{O}_{2}
$$

Since the equilibrium shifts to the right with temperature increase, the oxygen activity in the melt will increase. Such 


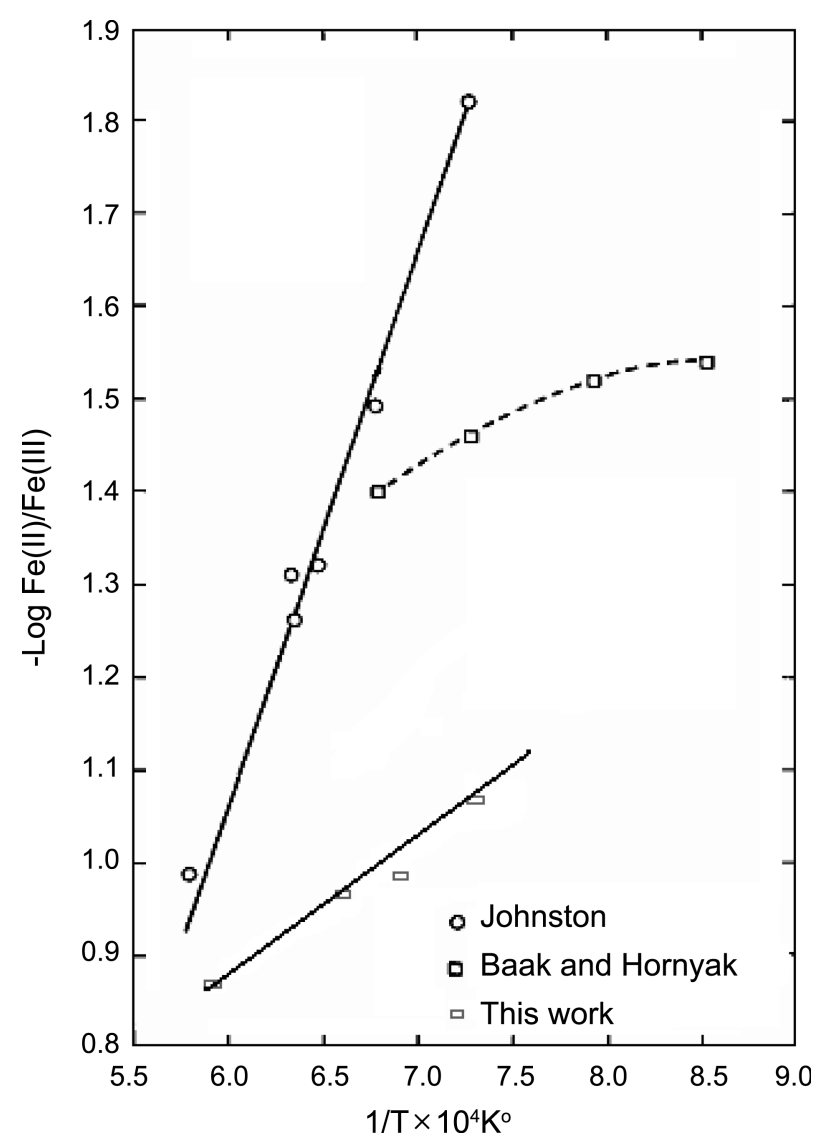

Fig. 4. Effect of increasing temperature on $\frac{\mathrm{Fe}^{2+}}{\mathrm{Fe}^{3+}}$ equilibrium ratio in iron-sodium silicate glasses.

phenomena are well known not only in glass ${ }^{6)}$ but also in melt containing redox species by oxygen equilibrium pressure ${ }^{13)}$ or voltammetry. ${ }^{14)}$ Nanoparticles of iron oxide in a batch cause the formation of the melt layer at low temperature. With increase of temperature, more oxygen is generated by the reduction of ferric ions; thus, more ferrous ions will be formed. ${ }^{6)}$ However, after the melt layer is formed on the batch during melting, the release of oxygen from the batch to the atmosphere is prevented due to the low diffusion coefficient of oxygen in the melt. Under such conditions, the reaction represented by equilibrium (1) is suppressed due to the high oxygen activity, and the concentration of $\mathrm{Fe}^{2+}$ in the melt is kept low. Also, with increase of temperature due to increasing oxygen ions, the existence of these ions enhances and consequently decreases oxygen activity in molten glass; therefore, the amount of ferrous ions increases. Finally, increase of temperature leads to the transformation of ferric ions to ferrous ions, and the intensity of ferrous peak in $1050 \mathrm{~nm}$ increases. Fig. 4 shows the effect of temperature increase on the $\frac{F e^{2+}}{F e^{3+}}$ equilibrium ratio in comparison with results of other studies. ${ }^{1,15)}$ The variation slope in Fig. 4 is due to the existence of nanoparticle iron oxide in the glass batch, which leads to rapid formation of the molten layer with batch powder. This phenomenon takes place at low temperature, and non-bridging oxygen cannot exit from the molten glass, and fewer ferrous ions are formed. This phenomenon is not possible when micro size iron powder is used because melt is maintained at higher temperatures to achieve an equilibrium condition. All the above suggestions based on the reactions in (1) are consistent with the $\mathrm{Fe}^{2+}$ to total-Fe ratios shown in Table 2 . We will now discuss the oxygen activity in the melt. As seen in Table 2, the $\mathrm{Fe}^{2+}$ to total-Fe ratios show good correlation. This suggests that the equilibrium expressed by (1) controls the concentrations of oxygen, $\mathrm{Fe}^{2+}$, and $\mathrm{Fe}^{3+}$ in the melts and that the $\mathrm{Fe}^{2+}$ to total-Fe ratio is maintained in the glass produced. However, the results suggest that the total amount of oxygen diffused into the melt from the atmosphere is not large enough to affect the $\mathrm{Fe}^{2+}$ to total-Fe ratio except in samples containing only a small amount of $\mathrm{Fe}_{2} \mathrm{O}_{3}$.

\section{Conclusion}

Sodium silicate glasses containing nanoparticle iron oxide as raw material were prepared by melting at various temperatures. The redox state of iron in the glass was measured by optical absorption spectroscopy and wet chemical analysis. The oxidation-reduction of iron was controlled by both batch melting temperature and nanoparticle iron oxide. Temperature increase led to reduction of ferric ions to ferrous ions. This resulted in the intensity increase of the ferrous peak at $1050 \mathrm{~nm}$ of the spectrum. The results of wet chemical analysis also showed an increase in the amount of ferrous ions with temperature increase. Introduction of nanoparticle iron oxide into the batch caused fewer ferrous ions to be formed, and the $\frac{F e^{2+}}{F e^{3+}}$ equilibrium ratio comparing with using micro-oxide iron powder decreased.

\section{Acknowledgments}

The authors are indebted to the ceramic laboratory of Qazvin University, which supplied the raw materials for the development of this research and to the Ceramic Department of Qazvin University for its financial support.

\section{REFERENCES}

1. W.D. Johnston, "Oxidation-reduction Equilibria in Ironcontaining Glass," J. Am. Ceram. Soc., 47 [47] 198-201 (1963).

2. W. Vogel, Properties of Colored Glasses; Vol. 1, pp. 138-200, Chemistry of Glass, New York, 1925.

3. F. V. Tolly, Foundation of Glass Industry; Vol. 3, pp. 13161318, Qazvin Glass Co, Qazvin, 1991.

4. C. R. Bamford, Color Generation and Control in Glass; Vol. 1, p. 36, Elsevier Scientific, New York, 1977.

5. K. Sakaguchi and T. Uchino, "Compositional Dependence of Infrared Absorption of Iron-doped Silicate Glasses," J. Non-Crystalline Solids., 353 [52-54] 4753-61 (2007). 
6. M. Yamashita, T. Akai, and R. Sawa, "Effect of Preparation Procedure on Redox States of Iron in Soda-lime Silicate Glass," J. Non-Crystalline Solids., 354 [40-41] 4534-38 (2008).

7. S.B. Donald, A.M. Swink, and H.D. Schreiber, "High-iron Ferric Glass," J. Non-Crystalline Solids., 352 [6-7] 539-43 (2006).

8. K.R. Kukadapu and G.L Li H.Smith, "Moossbauer and Optical Spectroscopic Study of Temperature and Redox Effects On Iron Local Environments in a Fe-doped (0.5 mol\% $\left.\mathrm{Fe}_{2} \mathrm{O}_{3}\right) 18 \mathrm{Na}_{2} \mathrm{O}-72 \mathrm{SiO}_{2}$ Glass," J. Non-Crystalline Solids., 317 [3] 301-18 (2003).

9. W.A. Weyl, Coloured Glasses; Vol. 1, pp. 60-89, Dawson's of Pall Mall, London, 1959.

10. C.R. Bamford, Glass Science and Technology, Colour Generation and Control in Glass; Vol. 2, p. 33, Elsevier Scientific Publishing Company, Amsterdam, 1977.

11. L.B. Globov, "Optical Absorption and Ionization of Silicate Glasses," J. Optical Materials., 4347 [1] 343-58 (2001).

12. J.S. Jeung and W Hoeard, "Effect of Oxidation State of Iron on Phase Separation in Sodium Silicate Glasses," J. Am. Ceram. Soc., 84 [8] 1859-64 (2001).

13. T. Tran and M.P. Brungs, "Applications of Oxygen Electrodes in Glassmelts: II, Oxygen Probes for the Measurement of Oxygen Potential in Sodium Disilicate Glass," J. Physics and Chemistry of Glasses., [21] 178-83 (1980).

14. Ki-Dong Kim and Sung-Ku Kwon, "Redox Equilibrium of $\mathrm{Fe}^{3+} / \mathrm{Fe}^{2+}$ and Diffusivity of Iron in Alkali-alkaline Earthsilicate Glass Melts," Journal of Ceramic Processing Research, 11 [4] 460-64 (2010).

15. J.E. Fenstermacher, "Optical Absorption Due to Tetrahedral and Octahedral Ferric Iron in Silicate Glasses," $J$. Non-Crystalline Solids., 38 [1] 239-44 (1980).

\section{Nomenclature}

nm nanometer

NIR near infrared

UV-Vis ultraviolet-visible 